\title{
Application of the geometrical theory of diffraction to Cassegrain subreflectors with laterally defocused feeds
}

Sørensen, O.; Rusch, W.

Published in:

I E E E Transactions on Antennas and Propagation

Publication date:

1975

Document Version

Publisher's PDF, also known as Version of record

Link back to DTU Orbit

Citation (APA):

Sørensen, O., \& Rusch, W. (1975). Application of the geometrical theory of diffraction to Cassegrain subreflectors with laterally defocused feeds. I E E E Transactions on Antennas and Propagation, 23(5), 698-702.

\section{General rights}

Copyright and moral rights for the publications made accessible in the public portal are retained by the authors and/or other copyright owners and it is a condition of accessing publications that users recognise and abide by the legal requirements associated with these rights.

- Users may download and print one copy of any publication from the public portal for the purpose of private study or research.

- You may not further distribute the material or use it for any profit-making activity or commercial gain

- You may freely distribute the URL identifying the publication in the public portal

If you believe that this document breaches copyright please contact us providing details, and we will remove access to the work immediately and investigate your claim. 

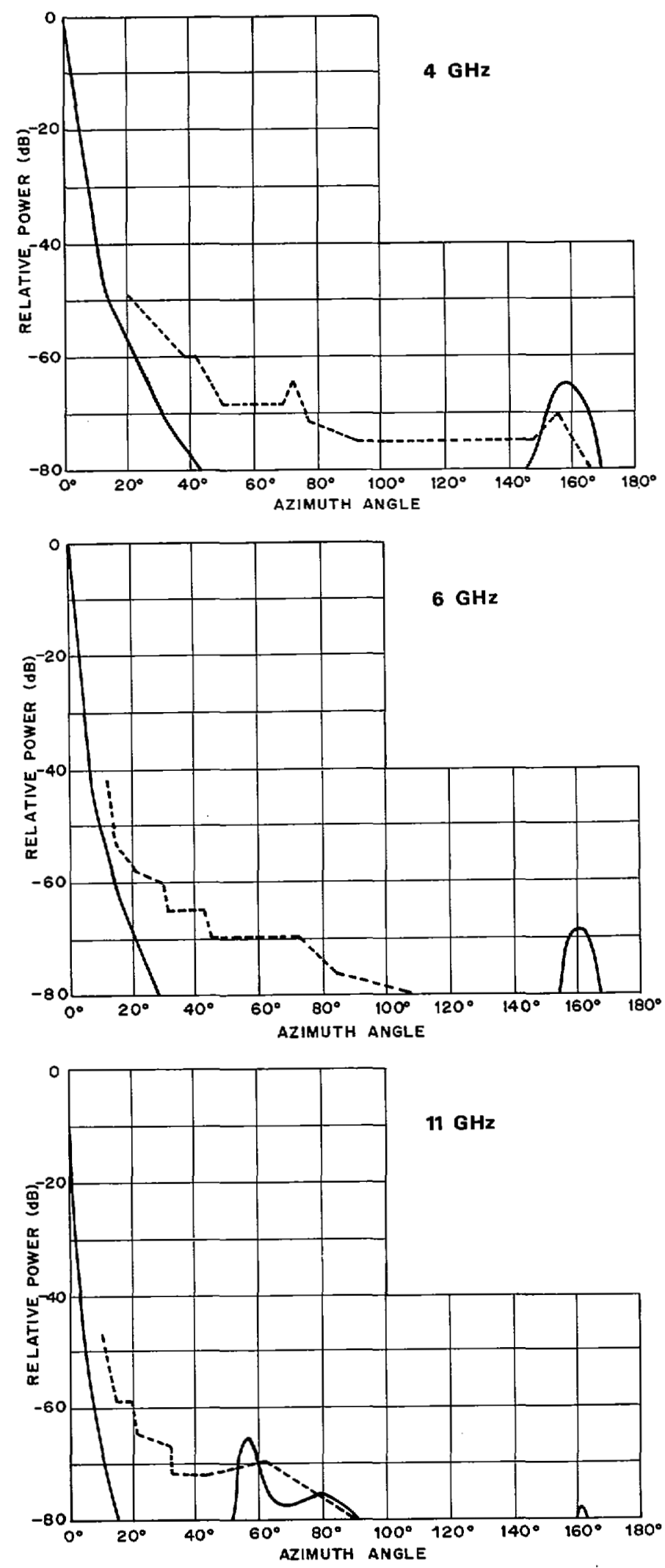

Fig. 7. Smoothed theoretical and measured radiation patterns of pyramidal horn reffector antenna with blinders in 4,6 , and $11 \mathrm{GHz}$ bands. GTD prediction - of the multiple edge blinders is, of course, to eliminate these high lobes.

To achieve grating lobe suppression at $11 \mathrm{GHz}$ necessitates the use of many edges. To clear the $p\left(\phi_{0}, \alpha, \beta_{c}\right)$ in Fig. 3 requires $N=17$ for values of $\beta_{c}$ larger than approximately $3^{\circ}$. From Fig. 5 , the major lobe at $4 \mathrm{GHz}$ is ideally eliminated by selecting values of $\delta$ and $\beta_{c}$ along the 17-edge contour. However, in view of the third observation in the design procedure, this is not particularly desirable. As a compromise, the angular combination $\beta_{c}=3.3^{\circ}$ (for a reasonable size blinder) and $\delta=19^{\circ}$ (to reduce the intensity of the major lobe) was chosen. Using the parameters $N=17, L=19$ in., $\beta_{c}=3.3^{\circ}, \alpha=14.5^{\circ}$, and $\delta=19^{\circ}$, the radiation patterns were theoretically predicted by GTD. These patterns are presented in Fig. 7. While prior experience has indicated that measured near-in sidelobes will be higher than theoretically predicted, there is a favorable disparity between GTD and measurements for grating and off-axis major lobes. The blinder was therefore built and tested.

Fig. 7 shows measured performance of the 17-edge blinder along with the respective GTD predictions. Note that experimentally observed major and grating lobes were lower than their predicted levels. Comparing these figures with Fig. 6 shows that in each case the $90^{\circ}$ sidelobe has been suppressed, and a general reduction in wide-angle sidelobes has been achieved. With the 17-edge blinders attached, this small fiber glass horn reflector provides excellent wide-angle azimuthal plane sidelobe suppression.

\section{ACKNOWLEDGMENT}

The author is pleased to acknowledge the contributions of the following individuals at Bell Laboratories: J. E. Richard for making the experimental antenna pattern measurements and P. E. Butzien and C. P. Bates for fruitful theoretical discussions they had with the author.

\section{REFERENCES}

[1] D. T. Thomas, "A half blinder for reducing certain sidelobes in large horn reflector antennas," IEEE Trans. Antennas Propagat. (Commun.), vol. AP-19, pp. 774-776, Nov. 1971.

[2] reflector antennas," Bell Syst. Tech. J., vol. 50, pp. 2979-2995, Nov.

[3] 1971. "Design of multiple-edge blinders for large horn reffector antennas," IEEE Trans. Antennas Propagat., vol. AP-21, pp. 153-158, Mar. 1973.

[4] C. A. Siller, Jr., "A fiber glass pyramidal horn reflector antenna for point-to-point microwave communications," in 1974 Int. IEEE/AP-S Symp., June 10-12, 1974.

\section{Application of the Geometrical Theory of Diffraction to Cassegrain Subreflectors with Laterally Defocused Feeds}

O. S $\phi R E N S E N$ AND W. V. T. RUSCH, SENIOR MEMBER, IEEE

Abstract-The geometrical theory of diffraction (GTD) as formulated by $R$. G. Kouyoumjian has been applied to predict the radiation characteristics of hyperboloidal subreflectors with laterally defocused feeds. In caustic or multicaustic directions the scattered fields are determined

Manuscript received October 10, 1974; revised March 17, 1975.

0 . S $\phi$ rensen is with the Electromagnetics Institute, Technical University of Denmark, Lyngby, Denmark.

W. V. T. Rusch is with the Electromagnetics Institute, Technical University of Denmark, Lyngby, Denmark, on leave from the Department of Electrical Engineering, University of Southern California, Los Angeles, Calif. 


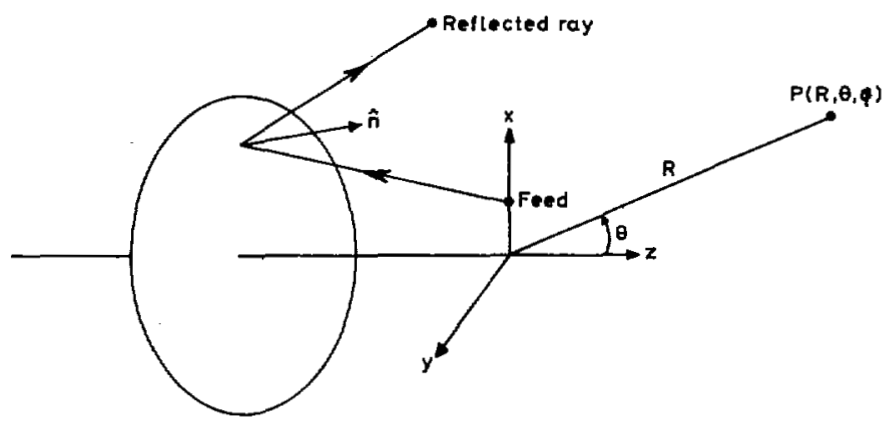

Fig. 1. Nonsymmetrically fed hyperboloid.

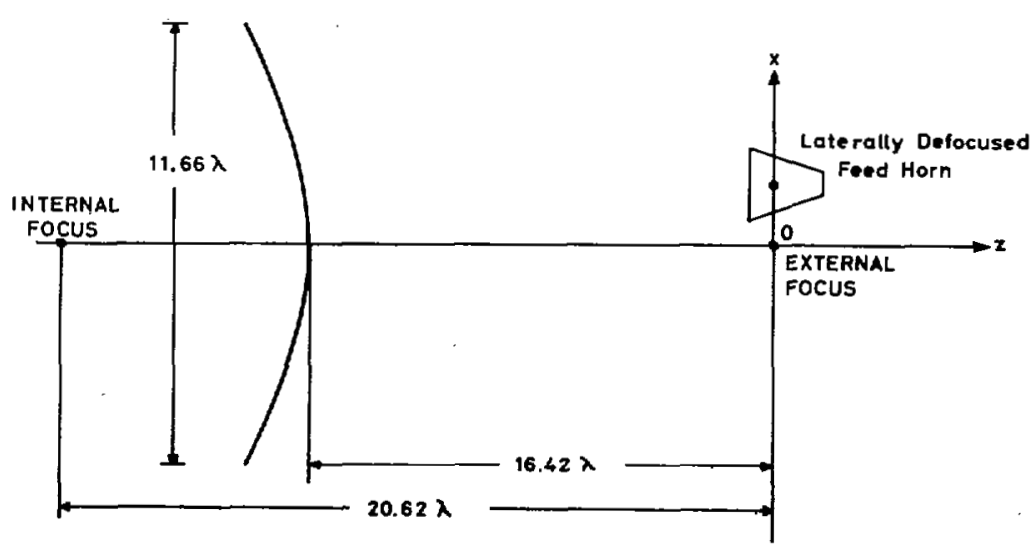

Fig. 2. Geometry of experimental setup.

using an equivalent ring current placed along the edge of the subreflector. The theoretical results are compared to measured amplitude and phase data. In order to improve the agreement, the blocking effects of the feed horn have been accounted for using the geometrical theory of diffraction. The calculated subreflector fields have been used to illuminate a paraboloid from which the scattered field is determined by physical optics. The results are compared to those obtained using a laterally defocused equivalent paraboloid.

\section{INTRODUCTION}

The ability to analyze Cassegrain antennas with laterally defocused feeds is necessary to predict the RF characteristics of a) Cassegrain monopulse antennas and b) Cassegrain antennas with feed systems which are unintentionally misaligned by unwanted mechanical or thermal forces. Lateral feed displacement destroys axial symmetry and makes the straightforward four-dimensional integral approaches prohibitively time-consuming [1]. The geometrical theory of diffraction (GTD) [2], which adapts easily to the symmetric Cassegrain antenna [3], can also be applied to the nonsymmetrical problem, although the analysis is significantly complicated by the oblique incidence of the rays at the subreflector edge.

\section{Application of GTD to the Laterally Defocused SUBREFLECTOR}

The geometry of the nonsymmetrically fed hyperboloid is illustrated in Fig. 1. The total field may be calculated by combining the specular (GO) ray (if one exists) with the edge contributions. The field of the specular ray is easily computed [4] once the specular point has been determined. This determination may be accomplished using a two-dimensional successive bisection method to solve Fermat's Law [5].

To calculate the diffracted field in a specified direction of the distant field point it is necessary to determine which points on the circular edge can be possible diffraction points. Molinet and Saltiel [6] have considered the problem in connection with the circular rim of a cylindrical satellite illuminated by an arbitrarily positioned point source and have found that there are a maximum of four possible diffraction points around the rim for a general direction of the distant field point.

Pontoppidan [7] has considered the special case of a circular edge and coplanar source point, field point, and reflector axis, for which there may be either two or four points of edge diffraction. However, for a general position of the field point, there is no simple criterion by which the number of points may be determined, and it becomes necessary to solve the problem numerically.

The two components of the far-zone field diffracted from a point $Q$ on the circular edge are related to the incident field components by [2]:

$$
\begin{array}{r}
{\left[\begin{array}{l}
E_{\beta_{0}}{ }^{d} \\
E_{\phi}{ }^{d}
\end{array}\right]=\left[\begin{array}{cc}
-D_{s} \sqrt{k} & 0 \\
0 & -D_{h} \sqrt{k}
\end{array}\right]\left[\begin{array}{cc}
\frac{1}{k} & E_{\beta_{0}}{ }^{i} \\
\frac{1}{k} & E_{\phi^{\prime}}{ }^{i}
\end{array}\right] \sqrt{k \rho_{c}}} \\
\cdot e^{j k\left(x_{Q} \sin \theta_{P} \cos \phi_{P}+y_{Q} \sin \theta_{P} \sin \phi_{P}+\left(z_{Q}+2 c\right) \cos \theta_{P}\right)}
\end{array}
$$

where $x_{Q}, y_{Q}, z_{Q}$ are the coordinates of $Q$ and $\theta_{P}$ and $\phi_{P}$ are the polar coordinates of the distant point $P$. The phase reference point is taken to be the hyperboloid's internal focus $(z=-2 c)$. (The factor $e^{-j k R} / R$ has been suppressed.) The soft and hard diffraction coefficients, $D_{s}$ and $D_{h}$, are defined in detail in [2]. Singularities at reflection and shadow boundaries are avoided by the inclusion of Fresnel integral transition functions. The caustic distance $\rho_{c}$ is given by

$$
\frac{1}{\rho_{c}}=\frac{1}{\rho_{e}^{i}}-\frac{\hat{n}_{e} \cdot\left(\hat{s}_{i}-\hat{s}_{d}\right)}{A \sin ^{2} \beta_{0}^{\prime}}
$$




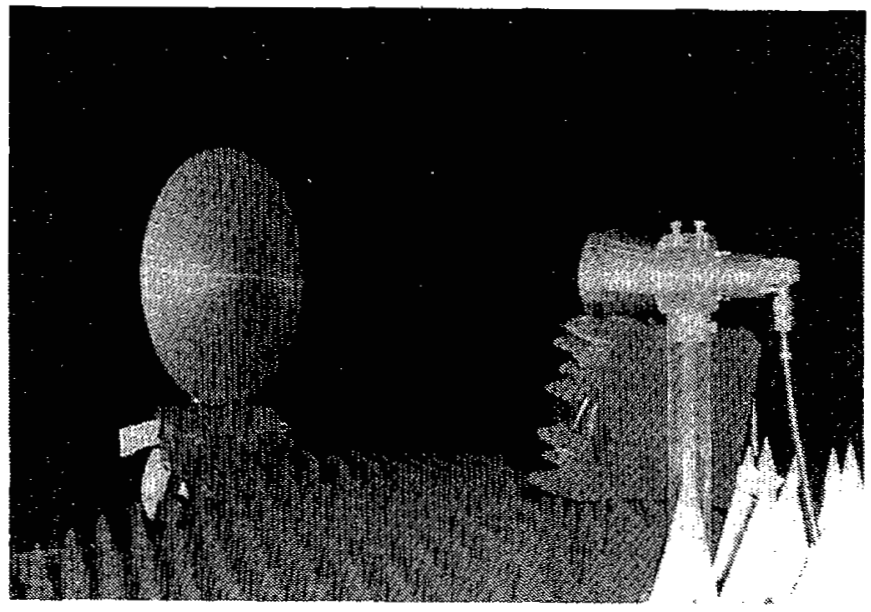

Fig. 3. Photograph of measurement setup.

where, for a point source, $\rho_{e}{ }^{i}$ is the distance from the source point to $Q, \hat{n}_{e}$ is the unit vector normal to the edge at $Q$ and directed away from the center of curvature, and $\hat{s}_{i}$ and $\hat{s}_{d}$ are unit vectors in the directions of incidence and diffraction, respectively. $A$ is the radius of the edge, and $\beta_{0}{ }^{\prime}$ is the angle between the incident ray and $\hat{t}$, the unit tangent to the edge at $Q$. The caustic distance is negative if the caustic lies between $Q$ and $P$.

The ordinary edge diffracted rays become unrealistically large in caustic or multicaustic directions. In such directions it is necessary to use equivalent electric and magnetic line sources placed along the edge to represent the fact that field contributions from many or all points on the edge are arriving at $P$ in phase synchronism or near synchronism. These currents are given by

$$
\begin{aligned}
& I_{e \phi} \cong\left(E_{\phi} D_{s} / \eta\right) \sqrt{(8 \pi / k)} e^{-j(\pi / 4)} \\
& I_{m \phi} \cong-H_{\phi} \eta D_{h} \sqrt{(8 \pi / k)} e^{-j(\pi / 4)}
\end{aligned}
$$

where $E_{\phi}$ and $H_{\phi}$ are the incident field components tangent to the edge, and $\eta$ is the impedance of free space. The Kouyoumjian group has evaluated the axial caustic fields of a focused paraboloid using these edge currents [8]. In the symmetric case it is possible to integrate the edge currents analytically and express the ring current field in terms of Bessel functions [9]. James and Kerdemelidis used the edge currents for the off-axis fields of a paraboloid [10]. In the present paper dealing with the laterally defocused hyperboloid, the edge currents have been used in caustic and multicaustic regions. The integrations are evaluated numerically, because the diffraction coefficients change as the integration variable varies along the edge of the reflector.

\section{EXPERIMENTAL RESULTS}

Fig. 2 shows the geometry of the experimental setup with an 11.7-wavelength-diameter hyperboloid illuminated by a $10-\mathrm{GHz}$ corrugated conical horn. A photograph of the setup is shown in Fig. 3. (Regrettably, this setup was somewhat sensitive to extraneous reflections from the supporting structure.) The hyperboloid's internal focus was placed on the axis of rotation of the antenna range. Amplitude and phase measurements were carried out both in the symmetric case and with the feed horn laterally displaced. Typical measured results are shown in Fig. 4(a) (amplitude) and 4(b) (phase) where they are compared with calculated fields based on GTD and edge currents (where necessary). As described in [9], the feed horn will block some GO rays and create an additional edge-diffracted ray in the planeof-scan, diffracted from the horn edge on the field-point-side of the axis. Furthermore, near the axial caustic it is necessary to use edge currents from those portions of the horn and reflector rims which are "visible" from the field point. These effects have been included in the calculated results, which generally agree to within a fraction of a $\mathrm{dB}$ and 5-10 electrical degrees of phase. Such accuracies are generally considered sufficient for Cassegrain calculations.

\section{Field Calculations for a lateraliy Defocused Cassegrann}

As discussed in the introduction, normal PO calculations become prohibitively time consuming for a laterally defocused Cassegrain antenna, because it is necessary to carry out a twodimensional integration over both reflectors. An alternative, is the use of a laterally defocused "equivalent paraboloid" [11]. A more accurate alternative, however, is the calculation of the subreflector field using GTD formulas and the use of this easily calculated field as the paraboloid illumination. With the exception of the first field point, this GTD calculation will require the same computer time as the equivalent paraboloid, while providing presumably the "true" illumination function.

Fig. 5 shows the scanned main beam and first sidelobes of a laterally defocused Cassegrain antenna (see figure for geometrical parameters) computed using both GTD and the equivalentparaboloid techniques for the hyperboloid fields and PO integration for the resulting paraboloid fields. Although the general features are substantially the same, the fields based on the GTD are generally $1-2 \mathrm{~dB}$ lower, because the proper paraboloid edge illumination taper has automatically been included in the calculation.

\section{Conclusions}

The geometrical theory of diffraction (GTD) has been used to analyze nonsymmetrically fed Cassegrain subreflectors. In caustic and multicaustic regions the scattered field has been calculated using equivalent electric and magnetic edge currents. The blocking effect of the horn has been accounted for by introducing an extra ray diffracted from the edge of the horn. In caustic regions, the edge currents have been used in connection with the leading edge of the feed horn. The calculations have been compared to measured amplitude and phase data. From these comparisons it can be concluded that GTD gives excellent results except in caustic regions where edge-current corrections can be used. (The remaining small discrepancies might be due either to experimental uncertainties or to the fact that the edgecurrent technique is not rigorous.) These GTD fields then provide accurate rapidly calculated illumination functions for the main paraboloidal reflector in laterally defocused Cassegrain antennas.

\section{REFERENCES}

[1] W. C. Wong, "On the equivalent parabola technique to predict the performance characteristics of a Cassegrain system with an offset feed," IEEE Trans. Antennas Propagat., vol. AP-21, pp. 335-339, May 1973 .

[2] R. G. Kouyoumjian and P. H. Pathak, "A uniform geometrical theory of diffraction for an edge in a perfectly conducting surface," Proc. IEEE, vol. 62, pp. 1448-1461, Nov. 1974.

[3] W. V. T. Rusch, "A comparison of geometrical and integral fields from high-frequency reflectors," Proc. IEEE, vol. 62, pp. 1603-1604, Nov. 1974.

[4] - "Reflector antennas," ch. 7, in Numerical Techniques for Antennas and Electromagnetics, R. Mittra, Ed. Berlin: Springer-Verlag, to be published in 1975 .

[5] $O$. S $\phi$ rensen, "Application of the geometrical theory of diffraction to Cassegrain subreflectors with laterally defocused feeds," Master's thesis, Electromagnetics Institute, Technical University of Denmark, Lyngby, Jan. 1974.

[6] F. Molinet and L. Saltiel, "High frequency radiation pattern prediction for satellite antennas," Laboratoire Central de Telecommunications, ESTEC Contract 1820/72 HP, July 1973.

[7] H. Bach, K. Pontoppidan, and L. Solymar, "A study of high frequency radiation pattern prediction for satellite antennas," Estec Contract $1821 / 72 \mathrm{HB}$, July 1973. 


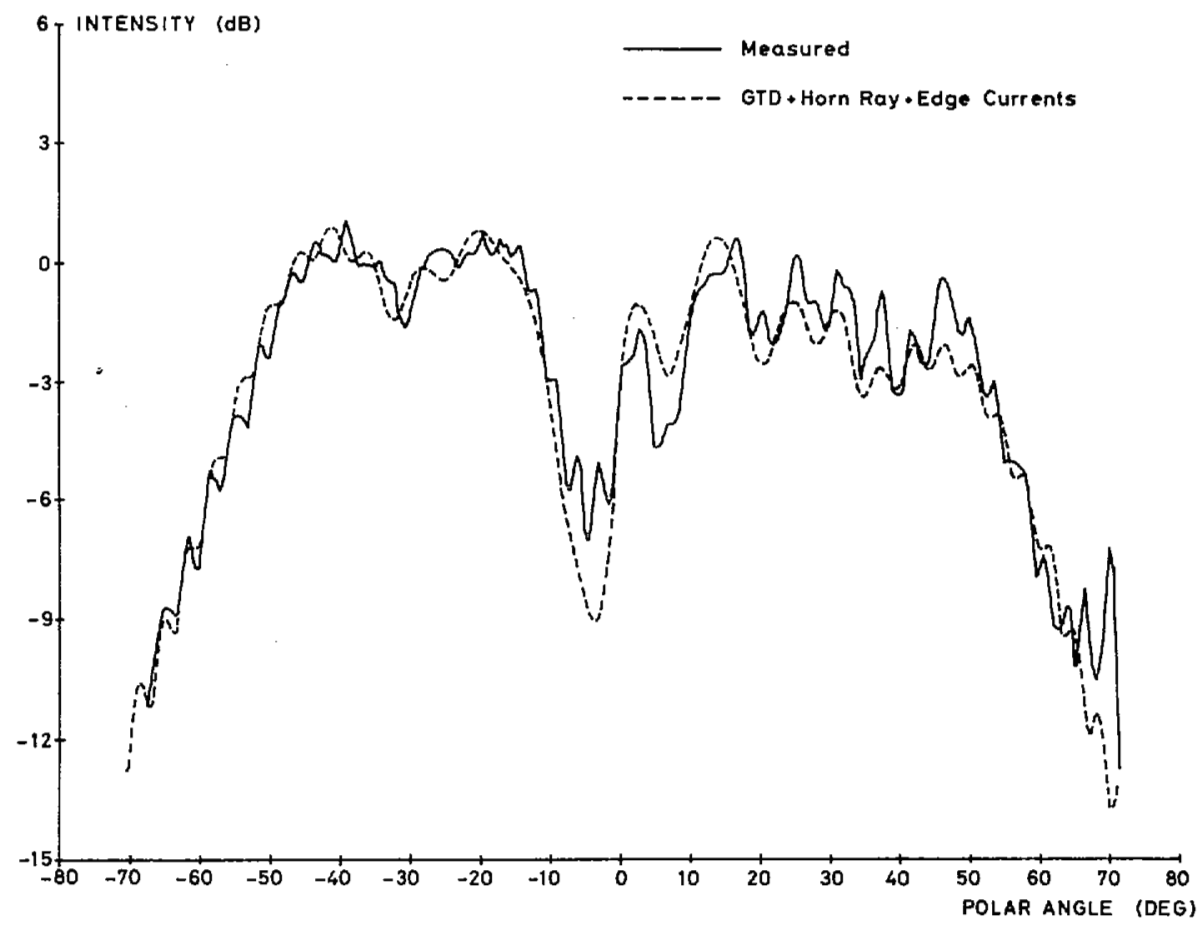

(a)

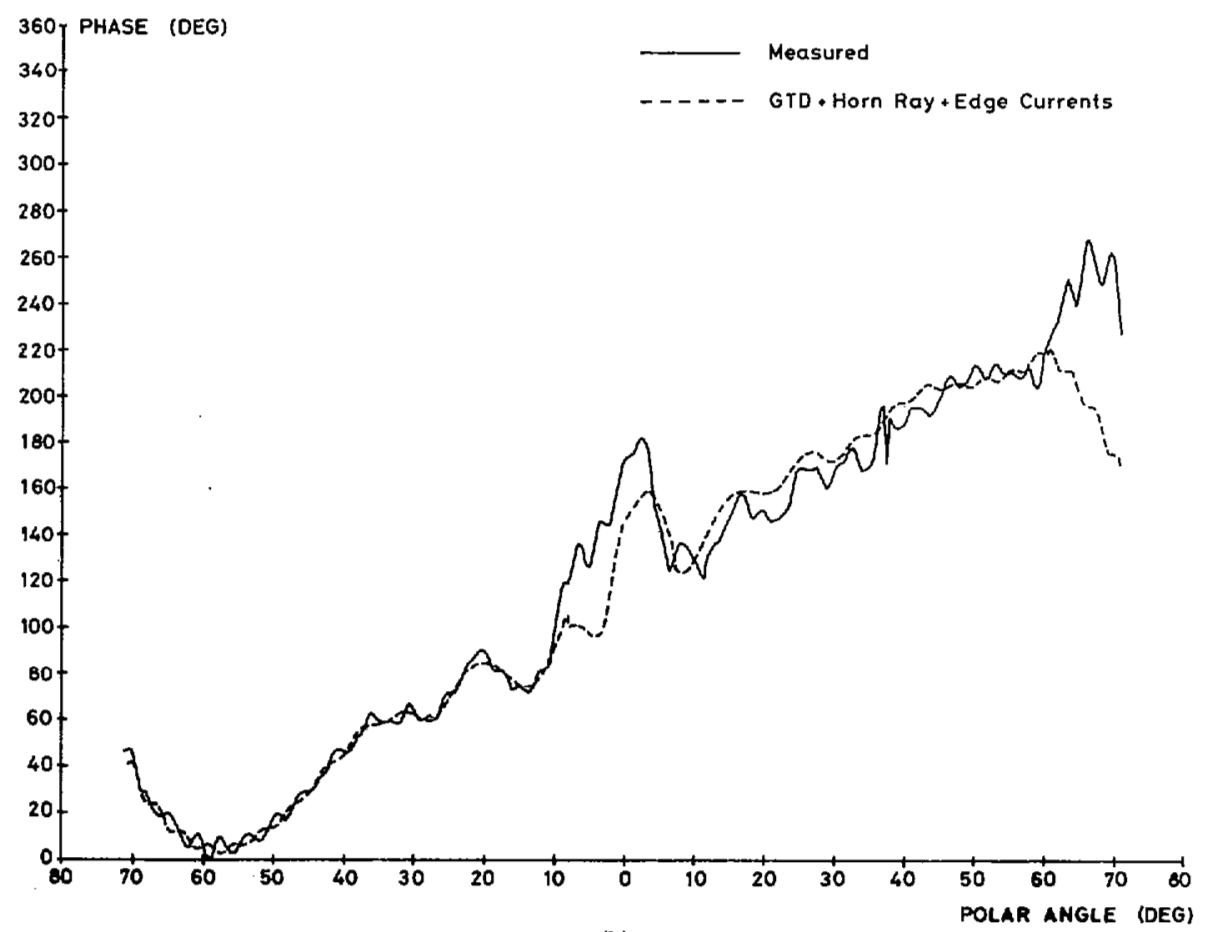

(b)

Fig. 4. (a) Comparison of computed and measured amplitude for one-wavelength laterally defocused hyperboloid. (b) Comparison of computed and measured phase for one-wavelength laterally defocused hyperboloid. 


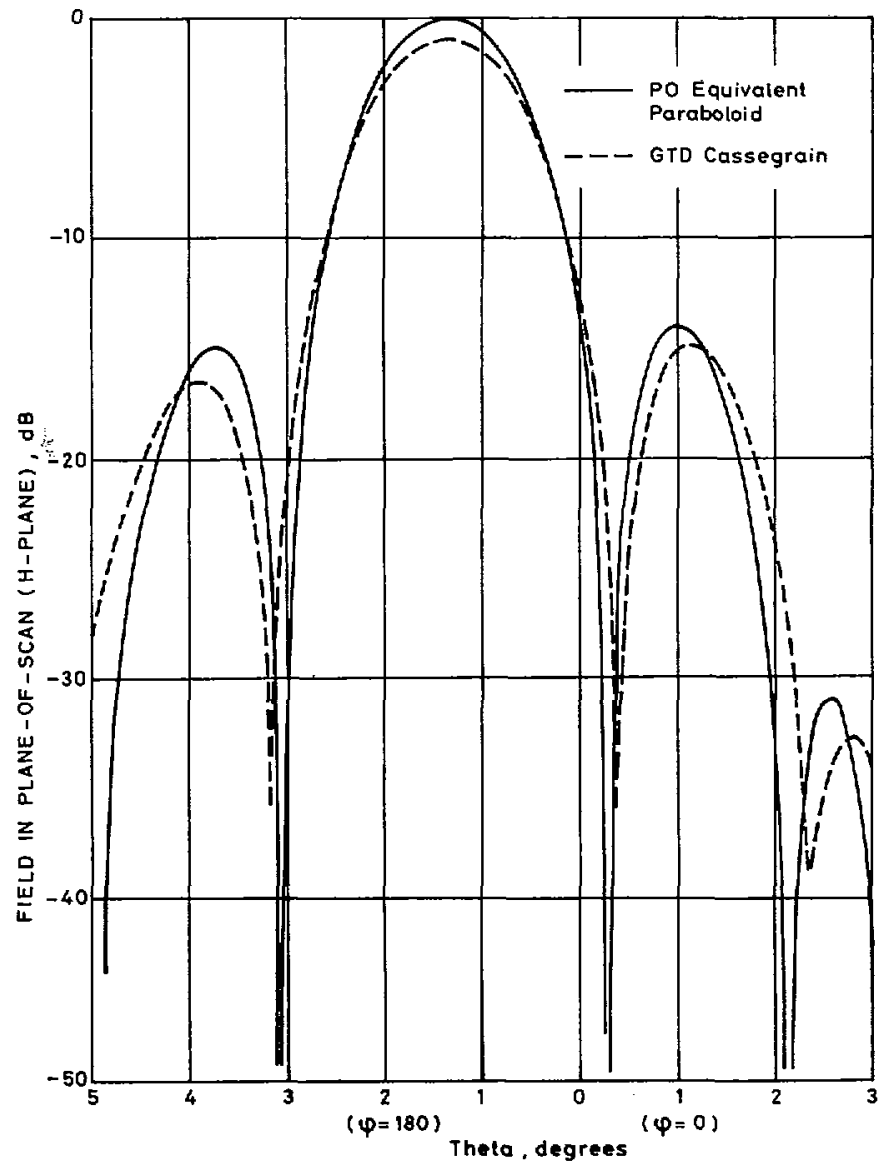

Fig. 5, Comparison of PO equivalent paraboloid and GTD Cassegrain for a Cassegrain antenna with $D_{\mathrm{par}}=40 \lambda, F / D=0.4, D_{\text {hyp }}=10 \lambda$, $e=2.29$, and $H$-plane scan of feed $=1 \lambda$.

[8] P. A. J. Ratnasiri, R. G. Kouyoumjian, and P. H. Pathak, "The wide angle sidelobes of refiector antennas," Ohio State Univ., Columbus, Tech. Rep. 2183-1, March 23, 1970.

[9] W. V. T. Rusch and O. Sørensen, "The geometrical theory of diffraction for axially symmetric reflectors," IEEE Trans. Anternas Propagat., vol. AP-23, pp. 414-419, May 1975 .

[10] G. L. James and V. Kerdemelidis, "Reflector antenna radiation analysis by equivalent edge currents," IEEE Trans. Antennas Propagat., yol. AP-21, pp. 19-24, Jan. 1973.

[11] P. W. Hannan, "Microwave antennas derived from the Cassegrain telescope," IRE Trans. Antennas Propagat., vol. AP-9, pp. 140-153, Mar. 1961.

\section{A GTD Analysis of the Far-Out Sidelobes of Cassegrain Antennas}

\section{A. MENTZER AND L. PETERS, JR.}

\begin{abstract}
Using recent developments of the geometrical theory of diffraction (GTD), the complete $E$ - and $H$-plane patterns of Cassegrain antennas are computed. The pattern in the main beam region is computed by conventional aperture integration methods and the GTD analysis is used to evaluate the far-out lobes caused by spillover and by various edge diffraction processes. The effects of the edge curvature and surface curvature are included in the computations.
\end{abstract}

Manuscript received October 7, 1974; revised April 21, 1975. This work was supported in part by Grant GK-33974 between the National Science Foundation, Washington, D.C. 20550, and the Ohio State University Research Foundation, Columbus, Ohio 43212.

C. A. Mentzer was with the Ohio State University Electro Science Laboratory, Department of Electrical Engineering, Columbus, Ohio. He is now with the Air Force Avionics Laboratory, Wright-Patterson Air Force Base, Ohio.

L. Peters, Jr., is with the Ohio State University ElectroScience Laboratory, Department of Electrical Engineering, Columbus, Ohio 43212.

\section{INTRODUCTION}

The complete patterns of only a few microwave pencil beam antennas have been computed, including horn antennas [1]-[4], center fed parabolic reflector antennas [5]-[7], offset fed parabolic reflector antennas [8], and horn-reflector antennas [9]. This paper focuses attention on the analysis of Cassegrain antenna systems. While there has been considerable work done on the effects of reflector shaping [10], polarization characteristics [11], efficiency [12], and general design [13], [14], little has been published about the far-out sidelobe levels of the Cassegrain. We will not examine one specific design which may or may not fit some "optimum" design, but rather establish general computational procedures to obtain the far-out sidelobe structure of Cassegrain antenna systems.

The analysis given follows the physical diffraction mechanisms involved and uses the geometrical theory of diffraction (GTD) to evaluate the fields associated with these diffractions. The edge diffracted fields of an infinite wedge may be associated with the incident field and with the field reflected from the surface. When the diffracting edge is the boundary of a curved surface, the incident field in the region where it is nonvanishing is not perturbed, but the reflected field in the region where it is nonvanishing is modified by the presence of the curved surface. Thus, the diffracted field component associated with this reflection is also altered. This change is accounted for by replacing the source (or caustic) by a virtual source (or caustic) which is the apparent source of the reflected field. This represents one of the basic modifications of the edge diffraction process introduced by Kouyoumjian and Pathak [15], [16].

The radiation pattern analysis of the Cassegrain is divided into two parts, the aperture integration for observation points near the main beam, and GTD for the sidelobe and backlobe region. The geometry of the antenna, shown in Fig. 1 is the most common Cassegrain design, namely a paraboloidal main reflector (focal length $=F_{p}$, diameter $=D_{p}$ ) and a hyperboloidal subreflector (focal length $=F_{H}$, diameter $=D_{H}$ ). One focus of the hyperboloid coincides with the focus of the paraboloid (located at $z=F_{p}$ ). The feed antenna, located at the other focus of the hyperboloid (at $z=F_{p}-2 F_{H}$ ), is assumed to have an axially symmetric pattern given by

$$
F(\theta, s)= \begin{cases}\left(0.00316+\cos ^{m} \theta\right) \frac{e^{-j k s}}{s}, & \text { for } \theta<90^{\circ} \\ (0.00316) \frac{e^{-j k s}}{s}, & \text { for } \theta \geq 90^{\circ}\end{cases}
$$

where $\theta$ is the polar angle, $s$ the range, and $m$ is chosen to provide the desired amplitude taper over the subreflector. The feed polarization is assumed to be such that the electric field is always parallel to the $x z$ plane. This feed pattern is used in determining the aperture fields used in the aperture integration and also in determining the spillover fields. This pattern function with a $-50 \mathrm{~dB}$ backlobe level is realistic for a feed antenna such as a corrugated horn. However any feed antenna pattern may be substituted for that given here and the remaining steps are unchanged.

\section{Main Beam Fields}

The portion of the pattern in the vicinity of the main beam is found by conventional aperture integration techniques. The aperture blockage due to the subreflector is treated by assuming an aperture distribution obtained from the negative value of the incident field after reflection by the parabolic surface taken over the shadow region of the hyperboloidal subreflector. These two sets of fields obtained by the aperture integrations include 